\title{
Minimax Estimation of Solutions of the First Order Linear Hyperbolic Systems with Uncertain Data
}

\author{
O. A. Kapustian *, O. G. Nakonechnyi , Yu. K. Podlipenko \\ Faculty of Computer Science and Cybernetics, Taras Shevchenko National University of Kyiv, Ukraine
}

\begin{abstract}
In this paper, we focus on optimal estimation of solutions of the Cauchy problem for the first order linear hyperbolic equation systems (or, more generally, estimation of values of some functionals on their solutions) under incomplete data.
\end{abstract}

Keywords Minimax estimation, First order hyperbolic system, Observation, Uncertain data.

AMS 2010 subject classifications: 35L56, 35Q60, 93C41.

DOI: $10.19139 /$ soic-2310-5070-457

\section{Introduction}

In this paper, we will solve the minimax estimation problem for solutions of the first order linear hyperbolic equation system under uncertain data.

Such problems appear in the frequent situation when the right-hand sides of the equations and initial conditions are not known exactly but only satisfy certain restrictions.

To solve the estimation problems we must have supplementary data (observations)

$$
y=C \varphi+\eta,
$$

where $\varphi$ is unknown solution, $C$ is an operator that specifies the method of measuring and $\eta$ is the measurement error. As a rule, this error is not known and belongs to a certain given set and the operator is not invertible. Therefore, in general, from given $y$, it is not possible to uniquely reconstruct the sought-for solution $\varphi$ and, consequently, quantity $l(\varphi)$, where $l$ is a given linear continuous functional. We see that a natural problem arises: to determine an estimate $\widehat{l(\varphi)}$ which would provide the best (in a certain sense) approximation to the sought-for $l(\varphi)$.

We assume that right-hand sides of equations and initial conditions are unknown and belong to the given bounded subsets of the space of all square integrable functions. It is supposed also that a class of noisy observations distributed on a finite system of bounded domains and observation errors (noises) are realizations of the stochastic processes, with unknown moment functions of the second order also belonging to certain given subsets.

To solve such problems with the lack of reliable information about the distribution of random perturbations, the following approach is used. We are looking for linear with respect to observations optimal estimates of solutions of the considered systems of equations from the condition of minimum of maximal mean square error of estimation taken over the above subsets. Such estimates are called minimax estimates.

*Correspondence to: Olena Kapustian (olena.kap@gmail.com), Taras Shevchenko National University of Kyiv, Faculty of Computer Science and Cybernetics, 60 Volodymyrska str., 01601 Kyiv, Ukraine

ISSN 2310-5070 (online) ISSN 2311-004X (print)

Copyright (C) 2019 International Academic Press 
This approach makes it possible to find optimal estimations of unknown solutions, corresponding to the "worst" cases of perturbations.

The above mentioned estimation problems are of major significance in many areas of applied physics - applied electromagnetics and acoustics. Therefore, comprehensive theoretical analysis of estimation techniques is an urgent task.

We elaborate constructive methods for obtaining such estimates, which is expressed in terms of solutions of special integro-differential equations.

Similar approach was applied for solving estimation problems for other types of ordinary and partial differential equations (see [5]-[13] and literature therein).

\section{Preliminaries and auxiliary results}

In this paper we denote matrices by bold capital letters and vectors by bold lower case letters. Denote by $H^{m}\left(\mathbb{R}^{n}\right)$ the set of all functions $f$ of $L^{2}\left(\mathbb{R}^{n}\right)$ whose derivatives up to the order $m$ (in the distribution sense) belong to $L^{2}\left(\mathbb{R}^{n}\right)$ and by $C^{m}([0, T] ; E)$ the set of all continuously differentiable functions $u(t)(t \in[0, T])$ with values in Hilbert space $E$.

Further, we will use the fact that for any $f, g \in H^{1}\left(\mathbb{R}^{n}\right)$ there holds

$$
\left(f, \frac{\partial}{\partial x_{i}} g\right)_{L^{2}\left(\mathbb{R}^{n}\right)}=-\left(\frac{\partial}{\partial x_{i}} f, g\right)_{L^{2}\left(\mathbb{R}^{n}\right)}, \quad i=1 \ldots, n,
$$

and for any functions $u, v \in C^{1}([0, T] ; E)$ the following integration by parts formula is valid:

$$
\int_{0}^{T}\left(\frac{d u(t)}{d t}, v\right)_{E} d t+\int_{0}^{T}\left(\frac{d v(t)}{d t}, u\right)_{E} d t=(u(T), v(T))_{E}-(u(0), v(0))_{E}
$$

where $(\cdot, \cdot)_{E}$ is the inner product in $E$ (see $\left.[2,3]\right)$.

Introduce the Hilbert space $L^{2}\left(\mathbb{R}^{n} \times(0, T)\right)^{N}$ with the inner product

$$
(\mathbf{u}, \mathbf{v})_{L^{2}\left(\mathbb{R}^{n} \times(0, T)\right)^{N}}=\int_{0}^{T} \int_{\mathbb{R}^{n}} \sum_{i=1}^{N} u_{i}(x, t) v_{i}(x, t) d x d t
$$

and the Hilbert space $L^{2}\left(0, T ;\left(H^{s}\left(\mathbb{R}^{n}\right)\right)^{N}\right)$ consisting of the functions of $t$ with values in the space $\left(H^{s}\left(\mathbb{R}^{n}\right)\right)^{N}$ and norm

$$
\|\mathbf{u}\|_{s}=\left(\int_{0}^{T}\|\mathbf{u}(t, \cdot)\|_{s}^{2} d t\right)^{1 / 2}
$$

where $\|\mathbf{f}\|_{s}$ is the norm of the vector-valued function $\mathbf{f}$ in the Sobolev space $\left(H^{s}\left(\mathbb{R}^{n}\right)\right)^{N}$.

Consider the first order hyperbolic system

$$
\frac{\partial \boldsymbol{\varphi}(x, t)}{\partial t}-\sum_{k=1}^{n} \mathbf{A}_{k}(x, t) \frac{\partial \boldsymbol{\varphi}(x, t)}{\partial x_{k}}-\mathbf{B}(x, t) \boldsymbol{\varphi}(x, t)=\mathbf{f}(x, t), \quad(x, t) \in \mathbb{R}^{n} \times(0, T),
$$

where

$$
\begin{gathered}
\mathbf{f}(x, t)=\left(f_{1}(x, t), \ldots, f_{N}(x, t)\right)^{T}, \\
\left.\varphi(x, t)=\boldsymbol{\varphi}(t)=\left(\varphi_{1}(x, t),\right) \ldots, \varphi_{N}(x, t)\right)^{T},
\end{gathered}
$$

$\mathbf{B}(x, t)=\left[b_{i j}(x, t)\right]$ is $N \times N$-matrix, $\mathbf{A}_{k}(x, t)=\left[a_{i j}^{(k)}(x, t)\right](k=1,2, \ldots, n)$ are symmetric matrices of order $N$, whose elements $b_{i j}(x, t), a_{i j}^{(k)}(x, t)$ are sufficiently smooth functions of $(x, t)$, bounded as well as their derivatives. 
By Friedrichs' theorem (see [1]), for any initial data $\varphi_{0} \in H^{1}\left(\mathbb{R}^{n}\right)^{N}$ and for $\mathbf{f} \in L^{2}\left(0, T ; H^{1}\left(\mathbb{R}^{n}\right)^{N}\right)$ there exsists a unique solution $\varphi$ of the system (1.3) such that $\varphi \in C^{0}\left([0, T] ; H^{1}\left(\mathbb{R}^{n}\right)^{N}\right) \cap C^{1}\left([0, T] ; L^{2}\left(\mathbb{R}^{n}\right)^{N}\right)$ and

$$
\varphi(x, 0)=\varphi_{0}(x) .
$$

Furthermore, there exists $C>0$ (independent of $\mathbf{f}$ and $\varphi_{0}$ ) such that for all $t \in[0, T]$

$$
\|\boldsymbol{\varphi}(\cdot, t)\|_{1}^{2} \leq C\left(\left\|\boldsymbol{\varphi}_{0}\right\|_{1}^{2}+\int_{0}^{t}\|\mathbf{f}(\tau)\|_{1}^{2} d \tau\right) .
$$

It follows immediately from Friedrichs' theorem that for any $\mathbf{g} \in L^{2}\left(0, T ; H^{1}\left(\mathbb{R}^{n}\right)^{N}\right)$ and $\psi_{0}(x) \in H^{1}\left(\mathbb{R}^{n}\right)^{N}$ the following problem formally adjoint to (1.3)-(1.4):

$$
\begin{aligned}
&-\frac{\partial \boldsymbol{\psi}(x, t)}{\partial t}+\sum_{k=1}^{n} \mathbf{A}_{k}(x, t) \frac{\partial \boldsymbol{\psi}(x, t)}{\partial x_{k}} \\
&+\left(\sum_{k=1}^{n} \frac{\partial \mathbf{A}_{k}(x, t)}{\partial x_{k}}-(\mathbf{B}(x, t))^{T}\right) \boldsymbol{\psi}(x, t)=\mathbf{g}, \quad(x, t) \in \mathbb{R}^{n} \times(0, T), \\
& \boldsymbol{\psi}(x, T)=\boldsymbol{\psi}_{0}(x), \quad x \in \mathbb{R}^{n}
\end{aligned}
$$

has a unique solution $\psi \in C^{0}\left([0, T] ; H^{1}\left(\mathbb{R}^{n}\right)^{N}\right) \cap C^{1}\left([0, T] ; L^{2}\left(\mathbb{R}^{n}\right)^{N}\right)$ and the following a priori estimate is valid

$$
\|\boldsymbol{\psi}(\cdot, t)\|_{1}^{2} \leq C\left(\left\|\boldsymbol{\psi}_{0}\right\|_{1}^{2}+\int_{0}^{T}\|\mathbf{g}(\tau)\|_{1}^{2} d \tau\right), \quad t \in[0, T]
$$

\section{Statement of the problem}

We now assume that in (1.3) and (1.4) the vector-functions $\mathbf{f}(x, t)$ and $\varphi_{0}(x)$ are unknown and belong to the set

$$
\begin{aligned}
G_{0}:=\left\{\tilde{\mathbf{F}}:=\left(\tilde{\mathbf{f}}, \tilde{\boldsymbol{\varphi}}_{0}\right) \in L^{2}\left(0, T ; H^{1}\left(\mathbb{R}^{n}\right)^{N}\right) \times H^{1}\left(\mathbb{R}^{n}\right)^{N}\right. & \\
& \begin{array}{r}
\int_{0}^{T} \int_{\mathbb{R}^{n}}\left(Q_{1}\left(\tilde{\mathbf{f}}-\mathbf{f}_{0}\right), \tilde{\mathbf{f}}-\mathbf{f}_{0}\right)_{\mathbb{R}^{N}} d x d t \\
\\
\left.\quad+\int_{\mathbb{R}^{n}}\left(Q_{2}\left(\tilde{\boldsymbol{\varphi}}_{0}-\boldsymbol{\varphi}_{0}^{(0)}\right), \tilde{\boldsymbol{\varphi}}_{0}-\boldsymbol{\varphi}_{0}^{(0)}\right)_{\mathbb{R}^{N}} d x \leq 1\right\},
\end{array}
\end{aligned}
$$

where $\quad \mathbf{f}_{0} \in L^{2}\left(0, T ; H^{1}\left(\mathbb{R}^{n}\right)^{N}\right), \quad \varphi_{0}^{(0)} \in H^{1}\left(\mathbb{R}^{n}\right)^{N}, \quad Q_{1}: L^{2}\left(0, T ; L^{2}\left(\mathbb{R}^{n}\right)^{N}\right) \rightarrow L^{2}\left(0, T ; L^{2}\left(\mathbb{R}^{n}\right)^{N}\right)$, $Q_{2}: L^{2}\left(\mathbb{R}^{n}\right)^{N} \rightarrow L^{2}\left(\mathbb{R}^{n}\right)^{N}$ are continuous positive definite selfadjoint operators, for which there exist bounded inverse operators.

Additionally, we suggest that $Q_{1}^{-1}$ and $Q_{2}^{-1}$ map the spaces $L^{2}\left(0, T ; H^{1}\left(\mathbb{R}^{n}\right)^{N}\right)$ and $H^{1}\left(\mathbb{R}^{n}\right)^{N}$ into themselves, respectively.

An estimation problem can be formulated as follows: from observations of the form

$$
\mathbf{y}_{i}(x, t)=\int_{0}^{T} \int_{D_{i}} \mathbf{K}_{i}(x, t, \xi, \tau) \varphi(\xi, \tau) d \xi d \tau+\boldsymbol{\eta}_{i}(x, t),(x, t) \in D_{i} \times(0, T) i=1, \ldots, m,
$$

of the state of system $\varphi$ described by Cauchy problem (1.3)-(1.4), to find the optimal (in a certain sense) estimate of the value of the functional

$$
l(\varphi):=\int_{0}^{T} \int_{D}\left(\mathbf{l}_{0}(x, t), \varphi(x, t)\right)_{\mathbb{R}^{N}} d x d t
$$


in the class of estimates

$$
\widehat{l(\boldsymbol{\varphi})}:=\sum_{i=1}^{m} \int_{0}^{T} \int_{D_{i}}\left(\mathbf{u}_{i}(x, t), \mathbf{y}_{i}(x, t)\right)_{\mathbb{R}^{N}} d x d \tau+c
$$

linear with respect to observations. Here $D_{i}, i=1, \ldots, m$, are bounded subdomains of $\mathbb{R}^{n}, D$ is subdomain which may coincide with $\mathbf{R}^{n}, \mathbf{K}_{i}(x, t, \xi, \tau)-N \times N$ is a matrix with elements belonging to $L^{2}\left(D_{i} \times(0, T)\right) \times L^{2}\left(D_{i} \times\right.$ $\left.(0, T)), \mathbf{l}_{0} \in L^{2}(0, T) ; H_{0}^{1}(D)^{N}\right)$ is a prescribed vector-function, $\mathbf{u}_{i} \in L^{2}\left(D_{i} \times(0, T)\right)^{N}, c \in \mathbb{R}, \mathbf{y}_{i}(x, t)=$ $\left(y_{i, 1}(x, t), \ldots, y_{i, N}(x, t)\right)^{T}$,

$\boldsymbol{\eta}_{i}(x, t)=\left(\eta_{i, 1}(x, t), \ldots, \eta_{i, N}(x, t)\right)^{T}$ are unknown random fields whose choice functions enter in observations (2.2) (hereafter the upper index $T$ denotes the transpose of a vector or matrix).

We suggest that

$$
\boldsymbol{\eta}:=\left(\boldsymbol{\eta}_{1}, \ldots, \boldsymbol{\eta}_{m}\right) \in G_{1},
$$

where by $G_{1}$ we denote the set of elements

$\tilde{\boldsymbol{\eta}}:=\left(\tilde{\boldsymbol{\eta}}_{1}, \ldots, \tilde{\boldsymbol{\eta}}_{m}\right)$ with components $\tilde{\boldsymbol{\eta}}_{i}(x, t)=\left(\tilde{\eta}_{i, 1}(x, t), \ldots, \tilde{\eta}_{i, N}(x, t)\right)^{T}$, which are random $\mathbb{R}^{N}$-valued functions defined in $D_{i} \times(0, T), i=1, \ldots m$, with integrable second moment functions $\mathbb{E}\left\|\tilde{\boldsymbol{\eta}}_{i}(x, t)\right\|_{\mathbb{R}^{n}}^{2}$ and zero expectations, satisfying the following conditions:

(i) random fields $\tilde{\boldsymbol{\eta}}_{i}(x, t)$ are pairwise uncorrelated, that is, mutually correlative matrices of fields $\tilde{\boldsymbol{\eta}}_{i}(x, t)$ and $\tilde{\boldsymbol{\eta}}_{j}(x, t)$ at $i \neq j$ are zero;

(ii) correlative matrices $\tilde{\mathbf{R}}_{i}(x, t, \xi, \tau)=\mathbb{E} \tilde{\boldsymbol{\eta}}_{i}(x, t)\left(\tilde{\boldsymbol{\eta}}_{i}(\xi, \tau)\right)^{T}$ of the fields $\tilde{\boldsymbol{\eta}}_{i}(x, t),(x, t),(\xi, \tau) \in D_{i} \times(0, T)$ $i=1, \ldots, m$, satisfy inequalities:

$$
\int_{0}^{T} \int_{D_{i}} \operatorname{Sp}\left[\tilde{\mathbf{Q}}_{i}(x, t) \tilde{\mathbf{R}}_{i}(x, t, x, t)\right] d x d t \leq 1, \quad i=1, \ldots, m,
$$

where $\tilde{\mathbf{Q}}_{i}(x, t)$ are positive definite $N \times N$-matrices with continuous in the domains $\bar{D}_{i}$ elements, and $\operatorname{Sp} \mathbf{S}=$ $\sum_{i=1}^{l} s_{i i}$ denotes the trace of the matrix $\mathbf{S}=\left\{s_{i j}\right\}_{i, j=1}^{l}$.

Besides, integral operators $\mathbf{P}_{i}$ with kernels $\left(\mathbf{K}_{i}(\xi, \tau, x, t)\right)^{T}$ defined according to

$$
\left(\mathbf{P}_{i} \psi\right)(x, t):=\int_{0}^{T} \int_{D_{i}}\left(\mathbf{K}_{i}(\xi, \tau, x, t)\right)^{T} \boldsymbol{\psi}(\xi, \tau) d \xi d \tau
$$

are assumed to be linear bounded operators acting from $L^{2}\left(D_{i} \times(0, T)\right)^{N}$ to $L^{2}\left(0, T ;\left(H_{0}^{1}\left(D_{i}\right)\right)^{N}\right), i=1, \ldots, m$. An example of such an operator is constructed in Appendix.

Introduce Hilbert space $\left.H:=L^{2}\left(D_{1} \times(0, T)\right)^{N} \times \ldots \times L^{2}\left(D_{m} \times(0, T)\right)^{N}\right)$ consisting of elements $\mathbf{u}:=$ $\left(\mathbf{u}_{1}, \ldots, \mathbf{u}_{m}\right)$ with components $\mathbf{u}_{i} \in L^{2}\left(D_{i} \times(0, T)\right)^{N}, i=1, \ldots, m$, with norm

$$
\|\mathbf{u}\|_{H}:=\left(\sum_{i=1}^{m}\left\|\mathbf{u}_{i}\right\|_{L^{2}\left(D_{i} \times(0, T)\right)^{N}}^{2}\right)^{1 / 2} .
$$

Definition. The estimate

$$
\widehat{\widehat{l(\boldsymbol{\varphi})}}=\sum_{i=1}^{m} \int_{0}^{T} \int_{D_{i}}\left(\hat{\mathbf{u}}_{i}^{(1)}(x, t), \mathbf{y}_{i}(x, t)\right)_{\mathbb{R}^{N}} d x d t+\hat{c}
$$

in which vector-functions $\hat{\mathbf{u}}_{i}(x, t)$ and a number $\hat{c}$ are determined from the condition

$$
\inf _{\mathbf{u} \in H, c \in \mathbb{R}} \sigma(\mathbf{u}, c)=\sigma(\hat{\mathbf{u}}, \hat{c}),
$$

where

$$
\sigma(u, c):=\sup _{\left(\tilde{f}, \tilde{\varphi}_{0}\right) \in G_{0}, \tilde{\boldsymbol{\eta}} \in G_{1}} \mathbb{E}[l(\tilde{\boldsymbol{\varphi}}),-\widehat{l(\tilde{\boldsymbol{\varphi}})}]^{2},
$$


$\tilde{\boldsymbol{\varphi}}$ is the solution to the problem (1.3)-(1.4) at $\mathbf{f}(x, t)=\tilde{\mathbf{f}}(x, t), \boldsymbol{\varphi}_{0}(x)=\tilde{\boldsymbol{\varphi}}_{0}(x)$,

$$
\widehat{l(\tilde{\boldsymbol{\varphi}})}=\sum_{i=1}^{m} \int_{0}^{T} \int_{D_{i}}\left(\mathbf{u}_{i}(x, t), \tilde{\mathbf{y}}_{i}(x, t)\right)_{\mathbb{R}^{N}} d x d t+c,
$$

and

$$
\tilde{\mathbf{y}}_{i}(x, t)=\int_{0}^{T} \int_{D_{i}} \mathbf{K}_{i}(x, t, \xi, \tau) \tilde{\boldsymbol{\varphi}}(\xi, \tau) d \xi d \tau+\tilde{\boldsymbol{\eta}}_{i}(x, t)
$$

will be called the minimax estimate of expression $l(\varphi)$.

The quantity

$$
\sigma=[\sigma(\hat{\mathbf{u}}, \hat{c})]^{1 / 2}
$$

will be called the error of the minimax estimation of (2.3).

Thus, the minimax estimate is an estimate minimizing the maximal mean-square estimation error calculated for the "worst" implementation of perturbations.

Note that the integrals in (2.8) and (2.10) which are understood as Lebesgue integrals exist with probability 1.

\section{Main results}

For every fixed $\mathbf{u}=\left(\mathbf{u}_{1}, \ldots, \mathbf{u}_{m}\right) \in H$ introduce a vector-function $\mathbf{z}(x, t ; \mathbf{u}) \in C^{0}\left([0, T] ; H^{1}\left(\mathbb{R}^{n}\right)^{N}\right) \cap$ $C^{1}\left([0, T] ; L^{2}\left(\mathbb{R}^{n}\right)^{N}\right)$ as a solution to the following initial value problem:

$$
\begin{gathered}
-\frac{\partial \mathbf{z}(x, t ; \mathbf{u})}{\partial t}+\sum_{k=1}^{n} \mathbf{A}_{k}(x, t) \frac{\partial \mathbf{z}(x, t ; \mathbf{u})}{\partial x_{k}}+\left(\sum_{k=1}^{n} \frac{\partial \mathbf{A}_{k}(x, t)}{\partial x_{k}}-(\mathbf{B}(x, t))^{T}\right) \mathbf{z}(x, t ; \mathbf{u}) \\
=\chi_{D}(x) \mathbf{l}_{0}(x, t)-\sum_{k=1}^{m} \chi_{D_{k}}(x) \int_{0}^{T} \int_{D_{k}}\left(\mathbf{K}_{k}(\xi, \tau, x, t)\right)^{T} \mathbf{u}_{k}(\xi, \tau) d \xi d \tau, \quad(x, t) \in \mathbb{R}^{n} \times(0, T), \\
\mathbf{z}(x, T ; \mathbf{u})=0, \quad x \in \mathbb{R}^{n}
\end{gathered}
$$

where $\chi_{M}(x)$, is a characteristic function of the set $M$. The function $\mathbf{z}(x, t ; \mathbf{u})$ is uniquely determined from equations (3.1) - (3.2). In fact, under our assumptions, the right-hand side of (3.1) equation belongs to $L^{2}\left(0, T ; H^{1}\left(\mathbb{R}^{n}\right)^{N}\right)$.

Lemma. Finding a minimax estimate of the value of functional $l(\varphi)$ is equivalent to the problem of optimal control of the system of integro-differential equation (3.1)-(3.2) with the cost function

$$
\begin{gathered}
I(\mathbf{u})=\int_{0}^{T} \int_{\mathbb{R}^{n}}\left(Q_{1}^{-1} \mathbf{z}(x, t ; \mathbf{u}), \mathbf{z}(x, t ; \mathbf{u})\right)_{\mathbb{R}^{N}} d x d t+\int_{\mathbb{R}^{n}}\left(Q_{2}^{-1} \mathbf{z}(x, 0 ; \mathbf{u}), \mathbf{z}(x, 0 ; \mathbf{u})\right)_{\mathbb{R}^{N}} d x \\
+\sum_{i=1}^{m} \int_{0}^{T} \int_{D_{i}}\left(\tilde{\mathbf{Q}}_{i}(x, t)^{-1} \mathbf{u}_{i}(x, t), \mathbf{u}_{i}(x, t)\right)_{\mathbb{R}^{N}} d x d t \rightarrow \min _{\mathbf{u} \in H} .
\end{gathered}
$$

Proof

Let $\tilde{\varphi}$ be a solution of the problem (1.3)-(1.4) at $\mathbf{f}(x, t)=\tilde{\mathbf{f}}(x, t), \boldsymbol{\varphi}(x)=\tilde{\varphi}_{0}(x)$. Then from relations (2.2)-(2.4), we obtain

$$
\begin{gathered}
l(\tilde{\boldsymbol{\varphi}})-\widehat{l(\tilde{\boldsymbol{\varphi}})}=\int_{0}^{T} \int_{D}\left(\mathbf{l}_{0}(x, t), \tilde{\boldsymbol{\varphi}}(x, t)\right)_{\mathbb{R}^{N}} d x d t \\
-\sum_{i=1}^{m} \int_{0}^{T} \int_{D_{i}}\left(\mathbf{u}_{i}(x, t), \tilde{\mathbf{y}}_{i}(x, t)\right)_{\mathbb{R}^{N}} d x d t-c
\end{gathered}
$$




$$
\begin{gathered}
\int_{0}^{T} \int_{D}\left(\mathbf{l}_{0}(x, t), \tilde{\boldsymbol{\varphi}}(x, t)\right)_{\mathbb{R}^{N}} d x d t-\sum_{i=1}^{m} \int_{D_{i}}\left(\mathbf{u}_{i}(x, t), \int_{0}^{T} \int_{D_{i}} \mathbf{K}_{i}(x, t, \xi, \tau) \tilde{\boldsymbol{\varphi}}(\xi, \tau) d \xi d \tau\right)_{\mathbb{R}^{N}} d x d t \\
-\sum_{i=1}^{m} \int_{0}^{T} \int_{D_{i}}\left(\mathbf{u}_{i}(x, t), \tilde{\boldsymbol{\eta}}_{i}(x, t)\right)_{\mathbb{R}^{N}} d x d t-c \\
=\int_{0}^{T} \int_{\mathbb{R}^{n}}\left(\chi_{D}(x) \mathbf{l}_{0}(x, t)-\sum_{i=1}^{m} \chi_{D_{i}}(x) \int_{0}^{T} \int_{D_{i}}\left(\mathbf{K}_{i}(\xi, \tau, x, t)\right)^{T} \mathbf{u}_{i}(\xi, \tau) d \xi d \tau, \tilde{\boldsymbol{\varphi}}(x, t)\right)_{\mathbb{R}^{N}} d x d t \\
-\sum_{i=1}^{m} \int_{0}^{T} \int_{D_{i}}\left(\mathbf{u}_{i}(x, t), \tilde{\boldsymbol{\eta}}_{i}(x, t)\right)_{\mathbb{R}^{N}} d x d t-c:=\xi .
\end{gathered}
$$

By Fubini's theorem we have, recalling that $\tilde{\boldsymbol{\eta}}_{i}(x, t), i=1, \ldots, m$, are vector processes with zero expectations,

$$
\mathbb{E}\left[\sum_{i=1}^{m} \int_{0}^{T} \int_{D_{i}}\left(\mathbf{u}_{i}(x, t), \tilde{\boldsymbol{\eta}}_{i}(x, t)\right)_{\mathbb{R}^{N}} d x d t\right]=\sum_{i=1}^{m}\left(\mathbf{u}_{i}, \mathbb{E} \tilde{\boldsymbol{\eta}}_{i}\right)_{L^{2}\left(D_{i}\right)^{N}}=0 .
$$

The latter equality and (3.4) yield

$$
\begin{aligned}
\mathbb{E}[l(\tilde{\boldsymbol{\varphi}})-\widehat{l(\tilde{\boldsymbol{\varphi}})}]=\int_{0}^{T} \int_{\mathbb{R}^{n}}\left(\chi_{D}(x) \mathbf{l}_{0}(x, t)\right. & \\
& \left.\quad-\sum_{i=1}^{m} \chi_{D_{i}}(x) \int_{0}^{T} \int_{D_{i}}\left(\mathbf{K}_{i}(\xi, \tau, x, t)\right)^{T} \mathbf{u}_{i}(\xi, \tau) d \xi d \tau, \tilde{\boldsymbol{\varphi}}(x, t)\right)_{\mathbb{R}^{N}} d x d t-c .
\end{aligned}
$$

From the equalities (3.4) and (3.5) we find, taking into consideration the known relationship $\mathbb{D} \xi=\mathbb{E} \xi^{2}-(\mathbb{E} \xi)^{2}$, that relates the variance $\mathbb{D} \xi=\mathbb{E}|\xi-\mathbb{E} \xi|^{2}$ of random variable $\xi$ to its expectation $\mathbb{E} \xi$, in which $\xi$ is determined by right-hand side of (3.4),

$$
\begin{gathered}
\mathbb{E}|l(\tilde{\boldsymbol{\varphi}})-\widehat{l(\tilde{\boldsymbol{\varphi}})}|^{2} \\
=\left|\int_{0}^{T} \int_{\mathbb{R}^{n}}\left(\chi_{D}(x) \mathbf{1}_{0}(x, t)-\sum_{i=1}^{m} \chi_{D_{i}}(x) \int_{0}^{T} \int_{D_{i}}\left(\mathbf{K}_{i}(\xi, \tau, x, t)\right)^{T} \mathbf{u}_{i}(\xi, \tau) d \xi d \tau, \tilde{\boldsymbol{\varphi}}(x, t)\right)_{\mathbb{R}^{N}} d x d t-c\right|^{2} \\
+\mathbb{E}\left|\sum_{i=1}^{m} \int_{0}^{T} \int_{D_{i}}\left(\mathbf{u}_{i}(x, t), \tilde{\boldsymbol{\eta}}_{i}(x, t)\right)_{\mathbb{R}^{N}} d x d t\right|^{2}
\end{gathered}
$$

Transform the first term in the right-hand side of (3.6). We get (see the explanations below)

$$
\begin{gathered}
\int_{0}^{T} \int_{\mathbb{R}^{n}}\left(\chi_{D}(x) \mathbf{l}_{0}(x)-\sum_{i=1}^{m} \chi_{D_{i}}(x) \int_{0}^{T} \int_{D_{i}}\left(\mathbf{K}_{i}(\xi, \tau, x, t)\right)^{T} \mathbf{u}_{i}(\xi, \tau) d \xi d \tau, \tilde{\boldsymbol{\varphi}}(x, t)\right)_{\mathbb{R}^{N}} d x d t \\
=\int_{0}^{T} \int_{\mathbb{R}^{n}}\left(-\frac{\partial \mathbf{z}(x, t ; \mathbf{u})}{\partial t}+\sum_{k=1}^{n} \mathbf{A}_{k}(x, t) \frac{\partial \mathbf{z}(x, t ; \mathbf{u})}{\partial x_{k}}\right. \\
\left.+\left(\sum_{k=1}^{n} \frac{\partial \mathbf{A}_{k}(x, t)}{\partial x_{k}}-(\mathbf{B}(x, t))^{T}\right) \mathbf{z}(x, t ; \mathbf{u}), \tilde{\boldsymbol{\varphi}}(x, t)\right)_{\mathbb{R}^{N}} d x d t \\
=\int_{0}^{T} \int_{\mathbb{R}^{n}}\left(-\frac{\partial \mathbf{z}(x, t ; \mathbf{u})}{\partial t}+\sum_{k=1}^{n} \frac{\partial}{\partial x_{k}}\left(\mathbf{A}_{k}(x, t) \mathbf{z}(x, t ; \mathbf{u})\right)-(\mathbf{B}(x, t))^{T} \mathbf{z}(x, t ; \mathbf{u}), \tilde{\boldsymbol{\varphi}}(x, t)\right)_{\mathbb{R}^{N}} d x d t
\end{gathered}
$$




$$
\begin{gathered}
\int_{0}^{T}\left(-\frac{\partial \mathbf{z}(\cdot, t ; \mathbf{u})}{\partial t}, \tilde{\varphi}(\cdot, t)\right)_{L^{2}\left(\mathbb{R}^{n}\right)^{N}} d t+\int_{0}^{T} \sum_{k=1}^{n} \sum_{i=1}^{N} \sum_{l=1}^{N}\left(\frac{\partial}{\partial x_{k}}\left(a_{i l}^{(k)}(\cdot, t) z_{l}(\cdot, t ; \mathbf{u})\right), \tilde{\varphi}_{i}(\cdot, t)\right)_{L^{2}\left(\mathbb{R}^{n}\right)} d t \\
-\int_{0}^{T} \int_{\mathbb{R}^{n}}\left((B(x, t))^{T} \mathbf{z}(x, t ; \mathbf{u}), \tilde{\boldsymbol{\varphi}}(x, t)\right)_{\mathbb{R}^{N}} d x d t \\
=\int_{0}^{T}\left(\frac{\partial \tilde{\boldsymbol{\varphi}}(x, t)}{\partial t}, \mathbf{z}(\cdot, t ; \mathbf{u})\right)_{L^{2}\left(\mathbb{R}^{n}\right)^{N}} d t-\int_{0}^{T} \sum_{k=1}^{n} \sum_{i=1}^{N} \sum_{l=1}^{N}\left(a_{i l}^{(k)}(\cdot, t) z_{l}(\cdot, t ; \mathbf{u}), \frac{\partial}{\partial x_{k}} \tilde{\varphi}_{i}(\cdot, t)\right)_{L^{2}\left(\mathbb{R}^{n}\right)} d t \\
-\int_{0}^{T} \int_{\mathbb{R}^{n}}(\mathbf{B}(x, t) \tilde{\boldsymbol{\varphi}}(x, t), \mathbf{z}(x, t ; \mathbf{u}))_{\mathbb{R}^{N}} d t+\int_{\mathbb{R}^{n}}(\tilde{\boldsymbol{\varphi}}(x, 0), \mathbf{z}(x, 0 ; \mathbf{u}))_{\mathbb{R}^{N}} d x \\
=\int_{0}^{T} \int_{\mathbb{R}^{n}}\left(\frac{\partial \tilde{\boldsymbol{\varphi}}(x, t)}{\partial t}-\sum_{k=1}^{n} \mathbf{A}_{k}(x, t) \frac{\partial \tilde{\boldsymbol{\varphi}}(x, t)}{\partial x_{k}}-\mathbf{B}(x, t) \tilde{\boldsymbol{\varphi}}(x, t), \mathbf{z}(x, t ; \mathbf{u})\right)_{\mathbb{R}^{N}} d x d t \\
\quad+\int_{\mathbb{R}^{n}}(\tilde{\boldsymbol{\varphi}}(x, 0), \mathbf{z}(x, 0 ; \mathbf{u}))_{\mathbb{R}^{N}} d x \\
\int_{0}^{T}(\tilde{\mathbf{f}}(x, t), \mathbf{z}(x, t ; \mathbf{u}))_{\mathbb{R}^{N}} d x d t+\int_{\mathbb{R}^{n}}\left(\tilde{\boldsymbol{\varphi}}_{0}(x), \mathbf{z}(x, 0 ; \mathbf{u})\right)_{\mathbb{R}^{N}} d x
\end{gathered}
$$

The first equality is valid due to the equation (3.1). The second, the third, and the fifth equalities are apparent. The fourth one holds as a result of applying the integration by parts formula (1.2) to the term $\int_{0}^{T}\left(-\frac{\partial \mathbf{z}(\cdot, t ; \mathbf{u})}{\partial t}, \tilde{\boldsymbol{\varphi}}(\cdot, t)\right)_{L^{2}\left(\mathbb{R}^{n}\right)^{N}} d t$, putting in this formula $E=L^{2}\left(\mathbb{R}^{n}\right)^{N}, u=$ $\mathbf{z}(\cdot, \cdot ; \mathbf{u}) \in C^{1}\left([0, T] ; L^{2}\left(\mathbb{R}^{n}\right)^{N}\right), v=\tilde{\varphi} \in C^{1}\left([0, T] ; L^{2}\left(\mathbb{R}^{n}\right)^{N}\right)$, and applying formula (1.1) to the expression $\left(\frac{\partial}{\partial x_{k}}\left(a_{i l}^{(k)}(\cdot, t) z_{l}(\cdot, t ; \mathbf{u})\right), \tilde{\varphi}_{i}(\cdot, t)\right)_{L^{2}\left(\mathbb{R}^{n}\right)}$, putting in it $f=a_{i l}^{(k)}(\cdot, t) z_{l}(\cdot, t ; \mathbf{u}) \in H^{1}\left(\mathbb{R}^{n}\right), g=\tilde{\varphi}_{i}(\cdot, t) \in H^{1}\left(\mathbb{R}^{n}\right)$ for any fixed $t \in(0, T)$. Finally, the sixth equality holds since $\tilde{\varphi}(x, t)$ is the solution of Cauchy problem (1.3)$(1.4)$ at $\mathbf{f}(x, t)=\tilde{\mathbf{f}}(x, t), \varphi_{0}(x)=\tilde{\varphi}_{0}(x)$.

Using relationships (3.6) and (3.7), we have

$$
\begin{aligned}
\mathbb{E}|l(\tilde{\boldsymbol{\varphi}})-\widehat{l(\tilde{\boldsymbol{\varphi}})}|^{2}= & \left|\int_{0}^{T}(\tilde{\mathbf{f}}(\cdot, t), \mathbf{z}(\cdot, t ; \mathbf{u}))_{L^{2}\left(\mathbb{R}^{n}\right)^{N}} d t+\left(\tilde{\boldsymbol{\varphi}}_{0}, \mathbf{z}(\cdot, 0 ; \mathbf{u})\right)_{L^{2}\left(\mathbb{R}^{n}\right)^{N}}-c\right|^{2} \\
& +\mathbb{E}\left|\sum_{i=1}^{m} \int_{0}^{T} \int_{D_{i}}\left(\mathbf{u}_{i}(x, t), \tilde{\boldsymbol{\eta}}_{i}(x, t)\right)_{\mathbb{R}^{N}} d x d t\right|^{2} \\
=\mid \int_{0}^{T}\left(\tilde{\mathbf{f}}(\cdot, t)-\mathbf{f}_{0}(\cdot, t), \mathbf{z}(\cdot, t ; \mathbf{u})\right)_{L^{2}\left(\mathbb{R}^{n}\right)^{N}} d t+\left(\tilde{\boldsymbol{\varphi}}_{0}-\boldsymbol{\varphi}_{0}^{0}, \mathbf{z}(\cdot, 0 ; \mathbf{u})\right)_{L^{2}\left(\mathbb{R}^{n}\right)^{N}} & \\
& +\int_{0}^{T}\left(\mathbf{f}_{0}(\cdot, t), \mathbf{z}(\cdot, t ; \mathbf{u})\right)_{L^{2}\left(\mathbb{R}^{n}\right)^{N}} d t+\left(\boldsymbol{\varphi}_{0}^{0}, \mathbf{z}(\cdot, 0 ; \mathbf{u})\right)_{L^{2}\left(\mathbb{R}^{n}\right)^{N}}-\left.c\right|^{2} \\
& +\sum_{i=1}^{m} \mathbb{E}\left|\int_{0}^{T} \int_{D_{i}}\left(\mathbf{u}_{i}(x, t), \tilde{\boldsymbol{\eta}}_{i}(x, t)\right)_{\mathbb{R}^{N}} d x d t\right|^{2}
\end{aligned}
$$

from which we obtain

$$
\inf _{c \in \mathbb{R}} \sup _{\left(\tilde{\mathbf{f}}, \tilde{\boldsymbol{\varphi}}_{0}\right) \in G_{0}, \tilde{\boldsymbol{\eta}} \in G_{1}} \mathbb{E}|l(\tilde{\boldsymbol{\varphi}})-\widehat{l(\tilde{\boldsymbol{\varphi}})}|^{2}=
$$




$$
\begin{aligned}
=\inf _{c \in \mathbb{R}} \sup _{\left(\tilde{\mathbf{f}}, \tilde{\boldsymbol{\varphi}}_{0}\right) \in G_{0}} \mid \int_{0}^{T}\left(\tilde{\mathbf{f}}(\cdot, t)-\mathbf{f}_{0}(\cdot, t), \mathbf{z}(\cdot, t ; \mathbf{u})\right)_{L^{2}\left(\mathbb{R}^{n}\right)^{N}} d t+\left(\tilde{\boldsymbol{\varphi}}_{0}-\boldsymbol{\varphi}_{0}^{0}, \mathbf{z}(\cdot, 0 ; \mathbf{u})\right)_{L^{2}\left(\mathbb{R}^{n}\right)^{N}} \\
\quad+\int_{0}^{T}\left(\mathbf{f}_{0}(\cdot, t), \mathbf{z}(\cdot, t ; \mathbf{u})\right)_{L^{2}\left(\mathbb{R}^{n}\right)^{N}} d t+\left(\boldsymbol{\varphi}_{0}^{0}, \mathbf{z}(\cdot, 0 ; \mathbf{u})\right)_{L^{2}\left(\mathbb{R}^{n}\right)^{N}}-\left.c\right|^{2} \\
+\sup _{\left(\tilde{\boldsymbol{\eta}}_{1}, \ldots, \tilde{\boldsymbol{\eta}}_{m}\right) \in G_{1}} \sum_{i=1}^{m} \mathbb{E}\left|\int_{0}^{T} \int_{D_{i}}\left(\mathbf{u}_{i}(x, t), \tilde{\boldsymbol{\eta}}_{i}(x, t)\right)_{\mathbb{R}^{N}} d x d t\right|^{2}
\end{aligned}
$$

From here, applying the generalized Schwarz's inequality (see, for example, [14]) together with inequalities (2.1) and (2.6), we find

$$
\inf _{c \in \mathbb{R}} \sup _{\left(\tilde{\mathbf{f}}, \tilde{\boldsymbol{\varphi}}_{0}\right) \in G_{0}, \tilde{\boldsymbol{\eta}} \in G_{1}} \mathbb{E}|l(\tilde{\boldsymbol{\varphi}})-\widehat{l(\tilde{\boldsymbol{\varphi}})}|^{2}=I(\mathbf{u}),
$$

where functional $I(\mathbf{u})$ is determined according to (3.3) and the infimum with respect to $c$ is attained at

$$
c=\int_{0}^{T}\left(\mathbf{f}_{0}(\cdot, t), \mathbf{z}(\cdot, t ; \mathbf{u})\right)_{L^{2}\left(\mathbb{R}^{n}\right)^{N}} d t+\left(\varphi_{0}^{0}, \mathbf{z}(\cdot, 0 ; \mathbf{u})\right)_{L^{2}\left(\mathbb{R}^{n}\right)^{N}} .
$$

The lemma is proved.

Further in the proof of theorem stated below, it will be shown that solving the optimal control problem (3.1)-(3.3) is reduced to solving some system of integro-differential equations.

Theorem 3.1. The minimax estimate of $l(\varphi)$ can be represented as

$$
\widehat{\widehat{l(\varphi)}}=\sum_{i=1}^{m} \int_{0}^{T} \int_{D_{i}}\left(\hat{\mathbf{u}}_{i}(x, t), \mathbf{y}_{i}(x, t)\right)_{\mathbb{R}^{N}} d x d t+\hat{c},
$$

where $\hat{c}=\int_{0}^{T} \int_{\mathbb{R}^{n}}\left(\hat{\mathbf{z}}(x, t), \mathbf{f}_{0}(x, t)\right)_{\mathbb{R}^{N}} d x d t+\int_{\mathbb{R}^{n}}\left(\hat{\mathbf{z}}(x, 0), \varphi_{0}^{(0)}(x)\right)_{\mathbb{R}^{N}} d x$

$$
\hat{\mathbf{u}}_{i}(x, t)=\tilde{\mathbf{Q}}_{i}(x, t) \int_{0}^{T} \int_{D_{i}} \mathbf{K}_{i}(x, t, \xi, \tau) \mathbf{p}(\xi, \tau) d \xi d \tau, \quad i=1, \ldots, m,
$$

and functions $\hat{\mathbf{z}}, \mathbf{p} \in C^{0}\left([0, T] ; H^{1}\left(\mathbb{R}^{n}\right)^{N}\right) \cap C^{1}\left([0, T] ; L^{2}\left(\mathbb{R}^{n}\right)^{N}\right)$ are determined from the integro-differential equation system

$$
\begin{gathered}
-\frac{\partial \hat{\mathbf{z}}(x, t)}{\partial t}+\sum_{k=1}^{n} \mathbf{A}_{k}(x, t) \frac{\partial \hat{\mathbf{z}}(x, t)}{\partial x_{k}}+\left(\sum_{k=1}^{n} \frac{\partial \mathbf{A}_{k}(x, t)}{\partial x_{k}}-(\mathbf{B}(x, t))^{T}\right) \hat{\mathbf{z}}(x, t) \\
=\chi_{D}(x) \mathbf{l}_{0}(x, t)-\sum_{k=1}^{m} \chi_{D_{k}}(x) \int_{0}^{T} \int_{D_{k}} \tilde{\mathbf{K}}_{k}\left(x, t, \xi_{1}, \tau_{1}\right) \mathbf{p}\left(\xi_{1}, \tau_{1}\right) d \xi_{1} d \tau_{1}, \quad(x, t) \in \mathbb{R}^{n} \times(0, T), \\
\hat{\mathbf{z}}(x, T)=0, \quad x \in \mathbb{R}^{n} \\
\frac{\partial \mathbf{p}(x, t)}{\partial t}-\sum_{k=1}^{n} \mathbf{A}_{k}(x, t) \frac{\partial \mathbf{p}(x, t)}{\partial x_{k}}-\mathbf{B}(x, t) p(x, t)=Q_{1}^{-1} \hat{\mathbf{z}}(x, t), \quad(x, t) \in \mathbb{R}^{n} \times(0, T) \\
\mathbf{p}(x, 0)=Q_{2}^{-1} \hat{\mathbf{z}}(x, 0), \quad x \in \mathbb{R}^{n}
\end{gathered}
$$


where ${ }^{\dagger}$

$$
\tilde{\mathbf{K}}_{k}\left(x, t, \xi_{1}, \tau_{1}\right)=\int_{0}^{T} \int_{D_{k}}\left(\mathbf{K}_{k}(\xi, \tau, x, t)\right)^{T} \tilde{\mathbf{Q}}_{k}(\xi, \tau) \mathbf{K}_{k}\left(\xi, \tau, \xi_{1}, \tau_{1}\right) d \xi d \tau
$$

Problem (3.10) - (3.13) is uniquely solvable. The error $\sigma$ of the minimax estimation of $l(\varphi)$ is given by the formula

$$
\sigma=[l(\mathbf{p})]^{1 / 2} .
$$

Proof

Taking into account estimate (1.8) one can easily verify that the funtional $I(\mathbf{u})$ is a strictly convex lower semicontinuous functional on $H$ and since

$$
I(\mathbf{u}) \geq \sum_{i=1}^{m} \int_{0}^{T} \int_{D_{i}}\left(\tilde{\mathbf{Q}}_{i}(x, t)^{-1} \mathbf{u}_{i}(x, t), \mathbf{u}_{i}(x, t)\right)_{\mathbb{R}^{N}} d x d t \geq c\|\mathbf{u}\|_{H}^{2} \quad \forall \mathbf{u} \in H, \text { c=const. }
$$

Then, by Remark 1.2 to Theorem 1.1 (see [4]), there exists one and only one element $\hat{\mathbf{u}}=\left(\hat{\mathbf{u}}_{1}, \ldots, \hat{\mathbf{u}}_{m}\right) \in H$ such that $I(\hat{\mathbf{u}})=\inf _{\mathbf{u} \in H} I(\mathbf{u})$.

Hence, for any fixed $\mathbf{w} \in H$ and $\tau \in \mathbb{R}$ the function $s(\tau):=I(\hat{\mathbf{u}}+\tau \mathbf{w})$ reaches its minimum at a unique point $\tau=0$, so that

$$
\left.\frac{d}{d \tau} I(\hat{\mathbf{u}}+\tau \mathbf{w})\right|_{\tau=0}=0
$$

Since $\mathbf{z}(x, t ; \hat{u}+\tau \mathbf{w})=\mathbf{z}(x, t ; \hat{u})+\tau \tilde{\mathbf{z}}(x, t ; \mathbf{w})$, where $\tilde{\mathbf{z}}(x, t ; \mathbf{w})$ is the unique solution to Cauchy problem (3.1)(3.2) at $\mathbf{u}=\mathbf{w}$ and $\mathbf{l}_{0}(x, t) \equiv 0$, from (3.3) and (3.15) we obtain

$$
\begin{gathered}
0=\left.\frac{1}{2} \frac{d I(\hat{\mathbf{u}}+\tau \mathbf{w})}{d \tau}\right|_{\tau=0} \\
=\lim _{\tau \rightarrow 0} \frac{1}{2 \tau}\left\{\int_{0}^{T} \int_{\mathbb{R}^{n}}\left[\left(Q_{1}^{-1} \mathbf{z}(x, t ; \hat{\mathbf{u}}+\tau \mathbf{w}), \mathbf{z}(x, t ; \hat{\mathbf{u}}+\tau \mathbf{w})\right)_{\mathbb{R}^{N}}-\left(Q_{1}^{-1} \mathbf{z}(x, t ; \hat{\mathbf{u}}), \tilde{\mathbf{z}}(x, t ; \hat{\mathbf{u}})\right)_{\mathbb{R}^{N}}\right] d x d t\right. \\
\quad \int_{\mathbb{R}^{n}}\left[\left(Q_{2}^{-1} \mathbf{z}(x, 0 ; \hat{\mathbf{u}}+\tau \mathbf{w}), \mathbf{z}(x, 0 ; \hat{\mathbf{u}}+\tau \mathbf{w})\right)_{\mathbb{R}^{N}}-\left(Q_{2}^{-1} \mathbf{z}(x, 0 ; \hat{\mathbf{u}}), \tilde{\mathbf{z}}(x, 0 ; \hat{\mathbf{u}})\right)_{\mathbb{R}^{N}}\right] d x \\
\left.+\sum_{i=1}^{m} \int_{0}^{T} \int_{D_{i}}\left[\left(\tilde{\mathbf{Q}}_{i}(x, t)^{-1}\left(\hat{\mathbf{u}}_{i}(x, t)+\mathbf{w}_{i}(x, t)\right), \hat{\mathbf{u}}_{i}(x, t)+\mathbf{w}_{i}(x, t)\right)_{\mathbb{R}^{N}}-\left(\tilde{\mathbf{Q}}_{i}(x, t)\right)^{-1} \hat{\mathbf{u}}_{i}(x, t), \hat{\mathbf{u}}_{i}(x, t)\right)_{\mathbb{R}^{N}}\right] d x d t \\
+\int_{\mathbb{R}^{n}}\left(Q_{1}^{-1} \mathbf{z}(x, t ; \hat{\mathbf{u}}), \tilde{\mathbf{z}}(x, t ; \mathbf{w})\right)_{\mathbb{R}^{N}} d x d t \\
\left.\quad Q_{2}^{-1} \mathbf{z}(x, 0 ; \hat{\mathbf{u}}), \tilde{\mathbf{z}}(x, 0 ; \mathbf{w})\right)_{\mathbb{R}^{N}} d x+\sum_{i=1}^{m} \int_{0}^{T} \int_{D_{i}}\left(\tilde{\mathbf{Q}}_{i}(x, t)^{-1} \hat{\mathbf{u}}_{i}(x, t), \mathbf{w}_{i}(x, t)\right)_{\mathbb{R}^{N}} d x d t
\end{gathered}
$$

Introduce function $\mathbf{p}(x, t) \in C^{0}\left([0, T] ; H^{1}\left(\mathbb{R}^{n}\right)^{N}\right) \cap C^{1}\left([0, T] ; L^{2}\left(\mathbb{R}^{n}\right)^{N}\right.$ as a unique solution to the initial value problem

$$
\frac{\partial \mathbf{p}(x, t)}{\partial t}-\sum_{k=1}^{n} \mathbf{A}_{k}(x, t) \frac{\partial \mathbf{p}(x, t)}{\partial x_{k}}-\mathbf{B}(x, t) p(x, t)=Q_{1}^{-1} \mathbf{z}(x, t ; \hat{\mathbf{u}}), \quad(x, t) \in \mathbb{R}^{n} \times(0, T),
$$

\footnotetext{
${ }^{\dagger}$ Here we use the following notation. If $\mathbf{A}(\xi)=\left[a_{i j}(\xi)\right]_{i, j=1}^{N}$ is a matrix depending on a variable $\xi$ belonging to a measurable set $\Omega$, then we define $\int_{\Omega} \mathbf{A}(\xi) d \xi$ by

$$
\int_{\Omega} \mathbf{A}(\xi) d \xi=\left[\int_{\Omega} a_{i j}(\xi) d \xi\right]_{i, j=1}^{N}
$$
}




$$
\mathbf{p}(x, 0)=Q_{2}^{-1} \mathbf{z}(x, 0 ; \hat{\mathbf{u}}), \quad x \in \mathbb{R}^{n} .
$$

Then the sum of the first two terms on the right-hand side of (3.16) can be written in the form

$$
\begin{gathered}
\int_{0}^{T} \int_{\mathbb{R}^{n}}\left(Q_{1}^{-1} \mathbf{z}(x, t ; \hat{\mathbf{u}}), \tilde{\mathbf{z}}(x, t ; \mathbf{w})\right)_{\mathbb{R}^{N}} d x d t+\int_{\mathbb{R}^{n}}\left(Q_{2}^{-1} \mathbf{z}(x, 0 ; \hat{\mathbf{u}}), \tilde{\mathbf{z}}(x, 0 ; \mathbf{w})\right)_{\mathbb{R}^{N}} d x \\
=\int_{0}^{T} \int_{\mathbb{R}^{n}}\left(\frac{\partial \mathbf{p}(x, t)}{\partial t}-\sum_{k=1}^{n} \mathbf{A}_{k}(x, t) \frac{\partial \mathbf{p}(x, t)}{\partial x_{k}}-\mathbf{B}(x, t) p(x, t), \tilde{\mathbf{z}}(x, t ; \mathbf{w})\right)_{\mathbb{R}^{N}} d x d t \\
\quad+\int_{\mathbb{R}^{n}}(\mathbf{p}(x, 0), \tilde{\mathbf{z}}(x, 0 ; \mathbf{w}))_{\mathbb{R}^{N}} d x \\
\int_{0}^{T} \int_{\mathbb{R}^{n}}\left(-\frac{\partial \tilde{\mathbf{z}}(x, t ; \mathbf{w})}{\partial t}+\sum_{k=1}^{n} \mathbf{A}_{k}(x, t) \frac{\partial \tilde{\mathbf{z}}(x, t ; \mathbf{w})}{\partial x_{k}}\right. \\
\left.\quad+\left(\sum_{k=1}^{n} \frac{\partial \mathbf{A}_{k}(x, t)}{\partial x_{k}}-(\mathbf{B}(x, t))^{T}\right) \tilde{\mathbf{z}}(x, t ; \mathbf{w}), \mathbf{p}(x, t)\right)_{\mathbb{R}^{N}} d x d t \\
=-\int_{0}^{T}\left(\sum_{k=1}^{m} \chi_{D_{k}} \int_{0}^{T} \int_{D_{k}}\left(\mathbf{K}_{k}\right)^{T}(\xi, \tau, \cdot, \cdot) \mathbf{w}_{k}(\xi, \tau) d \xi d \tau, \mathbf{p}\right)_{L^{2}\left(\mathbb{R}^{n}\right)^{N}} d t \\
=-\sum_{k=1}^{m} \int_{0}^{T} \int_{D_{k}}\left(\mathbf{w}_{k}(x, t), \int_{0}^{T} \int_{D_{k}} \mathbf{K}_{k}(x, t, \xi, \tau) \mathbf{p}(\xi, \tau) d \xi d \tau\right)_{\mathbb{R}^{N}} d x d t .
\end{gathered}
$$

From (3.19), (3.16), it follows that

$$
\begin{gathered}
\sum_{k=1}^{m} \int_{0}^{T} \int_{D_{k}}\left(\mathbf{w}_{k}(x, t), \int_{0}^{T} \int_{D_{k}} \mathbf{K}_{k}(x, t, \xi, \tau) \mathbf{p}(\xi, \tau) d \xi d \tau\right)_{\mathbb{R}^{N}} d x d t \\
\left.=\sum_{i=1}^{m} \int_{0}^{T} \int_{D_{i}}\left(\tilde{\mathbf{Q}}_{i}(x, t)\right)^{-1} \hat{\mathbf{u}}_{i}(x, t), \mathbf{w}_{i}(x, t)\right)_{\mathbb{R}^{N}} d x d t
\end{gathered}
$$

The last equality can be rewritten as

$$
\sum_{i=1}^{m}\left(\tilde{\mathbf{Q}}_{i}^{-1}\left[\hat{\mathbf{u}}_{i}-\tilde{\mathbf{Q}}_{i} \int_{0}^{T} \int_{D_{i}} \mathbf{K}_{i}(\cdot, \cdot, \xi, \tau) \mathbf{p}(\xi, \tau) d \xi d \tau\right], \mathbf{w}_{i}\right)_{L^{2}\left(D_{i} \times(0, T)\right)^{N}}=0 .
$$

$\forall \mathbf{w}=\left(\mathbf{w}_{1}, \ldots, \mathbf{w}_{m}\right) \in H$. Setting in $(3.20) \mathbf{w}_{i}=\hat{\mathbf{u}}_{i}-\tilde{\mathbf{Q}}_{i} \int_{0}^{T} \int_{D_{i}} \mathbf{K}_{i}(\cdot, \cdot, \xi, \tau) \mathbf{p}(\xi, \tau) d \xi d \tau, i=1, \ldots, m$, we get

$$
\begin{aligned}
\sum_{i=1}^{m}\left(\tilde{\mathbf{Q}}_{i}^{-1}\left[\hat{\mathbf{u}}_{i}-\tilde{\mathbf{Q}}_{i} \int_{0}^{T} \int_{D_{i}} \mathbf{K}_{i}(\cdot, \cdot, \xi, \tau) \mathbf{p}(\xi, \tau) d \xi d \tau\right]\right. & \\
& {\left.\left[\hat{\mathbf{u}}_{i}-\tilde{\mathbf{Q}}_{i} \int_{0}^{T} \int_{D_{i}} \mathbf{K}_{i}(\cdot, \cdot, \xi, d \tau) \mathbf{p}(\xi, \tau) d \xi d \tau\right]\right)_{L^{2}\left(D_{i} \times(0, T)\right)^{N}} }
\end{aligned}
$$

so that, by virtue of positive definiteness of the matrices $\tilde{\mathbf{Q}}_{i}^{-1}$,

$$
\hat{\mathbf{u}}_{i}=\tilde{\mathbf{Q}}_{i} \int_{0}^{T} \int_{D_{i}} \mathbf{K}_{i}(\cdot, \cdot, \xi, \tau) \mathbf{p}(\xi, \tau) d \xi d \tau, \quad i=1, \ldots, m
$$


Substituting the latter into (3.1), (3.2) and (3.8) and denoting $\hat{\mathbf{z}}(x, t)=\mathbf{z}(x, t ; \hat{\mathbf{u}})$, we see that $\hat{\mathbf{z}}(x, t)$ and $\mathbf{p}(x, t)$ satisfy system (3.10) - (3.13); the unique solvability of this system follows from the uniqueness of element $\hat{\mathbf{u}}$ of the functional (3.3).

Now let us establish that $\sigma=[l(\mathbf{p})]^{1 / 2}$. Substituting expressions (3.9) to (3.3), we obtain

$$
\begin{aligned}
& \sigma(\hat{\mathbf{u}}, \hat{c})= I(\hat{\mathbf{u}})=\int_{0}^{T} \int_{\mathbb{R}^{n}}\left(Q_{1}^{-1} \hat{\mathbf{z}}(x, t), \hat{\mathbf{z}}(x, t)\right)_{\mathbb{R}^{N}} d x d t+\int_{\mathbb{R}^{n}}\left(Q_{2}^{-1} \hat{\mathbf{z}}(x, 0), \hat{\mathbf{z}}(x, 0)\right)_{\mathbb{R}^{N}} d x \\
&+\sum_{i=1}^{m} \int_{0}^{T} \int_{D_{i}}\left(\left(\tilde{\mathbf{Q}}_{i}(x, t)\right)^{-1} \hat{\mathbf{u}}_{i}(x, t), \hat{\mathbf{u}}_{i}(x, t)\right)_{\mathbb{R}^{N}} d x d t \\
&= \int_{0}^{T} \int_{\mathbb{R}^{n}}\left(Q_{1}^{-1} \hat{\mathbf{z}}(x, t), \hat{\mathbf{z}}(x, t)\right)_{\mathbb{R}^{N}} d x d t+\int_{\mathbb{R}^{n}}\left(Q_{2}^{-1} \hat{\mathbf{z}}(x, 0), \hat{\mathbf{z}}(x, 0)\right)_{\mathbb{R}^{N}} d x \\
&+\sum_{i=1}^{m} \int_{0}^{T}\left(\tilde{\mathbf{Q}}_{i} \int_{D_{i}} \mathbf{K}_{i}(\cdot, \cdot, \xi, \tau) \mathbf{p}(\xi, \tau) d \xi d \tau, \int_{D_{i}} \mathbf{K}_{i}(\cdot, \cdot, \xi, \tau) \mathbf{p}(\xi, \tau) d \xi d \tau\right)_{L^{2}\left(D_{i}\right)^{N}}
\end{aligned}
$$

Transform the sum of the first and the second summands in the right-hand side of (3.22). Using relationships (3.9) - (3.13), we have

$$
\begin{gathered}
\int_{0}^{T} \int_{\mathbb{R}^{n}}\left(Q_{1}^{-1} \hat{\mathbf{z}}(x, t), \hat{\mathbf{z}}(x, t)\right)_{\mathbb{R}^{N}} d x d t+\int_{\mathbb{R}^{n}}\left(Q_{2}^{-1} \hat{\mathbf{z}}(x, 0), \hat{\mathbf{z}}(x, 0)\right)_{\mathbb{R}^{N}} d x \\
=\int_{0}^{T} \int_{\mathbb{R}^{n}}\left(\frac{\partial \mathbf{p}(x, t)}{\partial t}-\sum_{k=1}^{n} \mathbf{A}_{k}(x, t) \frac{\partial \mathbf{p}(x, t)}{\partial x_{k}}-\mathbf{B}(x, t) p(x, t), \hat{\mathbf{z}}(x, t)\right)_{\mathbb{R}^{N}} d x d t \\
+\int_{\mathbb{R}^{n}}(\mathbf{p}(x, 0), \hat{\mathbf{z}}(x, 0))_{\mathbb{R}^{N}} d x \\
\int_{0}^{T} \int_{\mathbb{R}^{n}}\left(-\frac{\partial \hat{\mathbf{z}}(x, t)}{\partial t}+\sum_{k=1}^{n} \mathbf{A}_{k}(x, t) \frac{\partial \hat{\mathbf{z}}(x, t)}{\partial x_{k}}+\left(\sum_{k=1}^{n} \frac{\partial \mathbf{A}_{k}(x, t)}{\partial x_{k}}-(\mathbf{B}(x, t))^{T}\right) \hat{\mathbf{z}}(x, t), \mathbf{p}(x, t)\right)_{\mathbb{R}^{N}} d x d t \\
=\int_{0}^{T}\left(\chi_{D} \mathbf{l}_{0}-\sum_{k=1}^{m} \chi_{D_{k}} \int_{D_{k}}\left(\mathbf{K}_{k}(\xi, \tau, \cdot, \cdot)\right)^{T} \hat{\mathbf{u}}_{k}(\xi, \tau) d \xi d \tau, \mathbf{p}\right)_{\mathbb{L}^{2}\left(\mathbb{R}^{n}\right)^{N}} \\
=\int_{0}^{T} \int_{D}\left(\mathbf{l}_{0}(x, t), \mathbf{p}(x, t)\right)_{\mathbb{R}^{N}} d x d t-\int_{0}^{T} \sum_{k=1}^{m}\left(\int_{D_{k}}\left(\mathbf{K}_{k}\right)^{T}(\xi, \tau, \cdot, \cdot) \hat{\mathbf{u}}_{k}(\xi, \tau) d \xi d \tau, \mathbf{p}\right)_{\mathbb{L}^{2}\left(D_{k}\right)^{N}} \\
=l(\mathbf{p})-\int_{0}^{T} \sum_{k=1}^{m}\left(\hat{\mathbf{u}}_{k}, \int_{D_{k}} \mathbf{K}_{k}(\cdot, \cdot, \xi, \tau) \mathbf{p}(\xi, \tau) d \xi d \tau\right)_{\mathbb{L}^{2}\left(D_{k}\right)^{N}} d t \\
=l(\mathbf{p})-\int_{0}^{T} \sum_{i=1}^{m}\left(\tilde{\mathbf{Q}} \int_{D_{i}} \mathbf{K}_{i}(\cdot, \cdot, \xi, \tau) \mathbf{p}(\xi, \tau) d \xi d \tau, \int_{D_{i}} \mathbf{K}_{i}(\cdot, \cdot, \xi, \tau) \mathbf{p}(\xi, \tau) d \xi d \tau\right)_{\left(L^{2}\left(D_{i}\right)\right)^{N}}(3.23)
\end{gathered}
$$

Equality (3.14) follows now from two relationships (3.23) and (3.22).

An alternative representation for the minimax estimate of $l(\varphi)$ in terms of the solution to a system of integrodifferential equations is given in the next theorem. This solution is independent of the specific form of functional (2.3). 
Theorem 3.2. The minimax estimate of (2.3) has the form

$$
\widehat{\widehat{l(\boldsymbol{\varphi})}}=l(\hat{\boldsymbol{\varphi}})=\int_{0}^{T} \int_{D}\left(\mathbf{l}_{0}(x, t), \hat{\boldsymbol{\varphi}}(x, t)\right)_{\mathbb{R}^{N}} d x d t
$$

where function $\hat{\varphi} \in C^{0}\left([0, T] ; H^{1}\left(\mathbb{R}^{n}\right)^{N}\right) \cap C^{1}\left([0, T] ; L^{2}\left(\mathbb{R}^{n}\right)^{N}\right)$ is determined from the solution to the problem $(3.25)-(3.28)$ :

$$
\begin{gathered}
-\frac{\partial \hat{\mathbf{p}}(x, t)}{\partial t}+\sum_{k=1}^{n} \mathbf{A}_{k}(x, t) \frac{\partial \hat{\mathbf{p}}(x, t)}{\partial x_{k}}+\left(\sum_{k=1}^{n} \frac{\partial \mathbf{A}_{k}(x, t)}{\partial x_{k}}-(\mathbf{B}(x, t))^{T}\right) \hat{\mathbf{p}}(x, t) \\
=\mathbf{d}(x, t)-\sum_{k=1}^{m} \chi_{D_{k}}(x) \int_{0}^{T} \int_{D_{k}} \tilde{\mathbf{K}}_{k}\left(x, t, \xi_{1}, \tau_{1}\right) \hat{\boldsymbol{\varphi}}(x, t) d \xi_{1} d \tau_{1}, \quad(x, t) \in \mathbb{R}^{n} \times(0, T), \\
\hat{\mathbf{p}}(x, T)=0, \quad x \in \mathbb{R}^{n}, \\
\frac{\partial \hat{\boldsymbol{\varphi}}(x, t)}{\partial t}-\sum_{k=1}^{n} \mathbf{A}_{k}(x, t) \frac{\partial \hat{\boldsymbol{\varphi}}(x, t)}{\partial x_{k}}-\mathbf{B}(x, t) \hat{\boldsymbol{\varphi}}(x, t) \quad=Q_{1}^{-1} \hat{\mathbf{p}}(x, t)+\mathbf{f}_{0}(x, t), \quad(x, t) \in \mathbb{R}^{n} \times(0, T), \\
\hat{\boldsymbol{\varphi}}(x, 0)=Q_{2}^{-1} \hat{\mathbf{p}}(x, 0)+\boldsymbol{\varphi}_{0}^{0}(x), \quad x \in \mathbb{R}^{n},
\end{gathered}
$$

where

$$
\begin{gathered}
\hat{\mathbf{p}} \in C^{0}\left([0, T] ; H^{1}\left(\mathbb{R}^{n}\right)^{N}\right) \cap C^{1}\left([0, T] ; L^{2}\left(\mathbb{R}^{n}\right)^{N}\right), \\
\mathbf{d}(x, t)=\sum_{i=1}^{m} \chi_{D_{i}}(x) \int_{0}^{T} \int_{D_{i}}\left(\mathbf{K}_{i}(\xi, \tau, x, t)\right)^{T} \tilde{\mathbf{Q}}_{i}(\xi, \tau) \mathbf{y}_{i}(\xi, \tau) d \xi d \tau
\end{gathered}
$$

and the right-hand side in (3.25) is considered for every realization of random functions $\boldsymbol{\eta}_{i}(\xi, \tau)$ entering into $\mathbf{y}_{i}(\xi, \tau)$ which belong with probability 1 to the space $L^{2}\left(D_{i} \times(0, T)\right), i=1, \ldots, m$. Problem (3.25)-(3.28) is uniquely solvable.

The random fields $\hat{\mathbf{p}}(x, t)$ and $\hat{\varphi}(x, t)$, whose realizations satisfy problem (3.25)-(3.28), possess Lebesgue integrable second moments.

Proof

The proof of this theorem is similar to the proof of Theorem 3.1.

Remark 1. Function $\hat{\varphi}(x, t)$ which is determined from the solution to problem (3.25)-(3.28) can be taken as a good estimate (in a certain sense) of the unknown solution $\varphi(x, t)$ to the Cauchy problem (1.3)-(1.5).

Remark 2. Using the decoupling technique by J.-L. Lions [4] solving each of the systems of integro-differential equations (3.10) - (3.13) and (3.25)-(3.28) can be reduced to solving a nonlinear operator Riccati equation with the condition at $t=T$ and a first order linear hyperbolic system with the initial conditions at $t=0$.

\section{Conclusions}

In this paper, the minimax estimation problem for solutions of the first order linear hyperbolic equation system with uncertain data is solved. It was shown that the finding a minimax estimate is equivalent to the corresponding optimal control problem for the system of integro-differential equations. The minimax estimate and the alternative representation for this estimate from the solution to the system of integro-differential equations are obtained. An example of observation operator is constructed in Appendix. 


\section{Appendix}

Here we give an example of an observation operator, for which the operator (2.2) satisfies the conditions listed on page 698. Let in the observation operator (2.2) the matrix $\mathbf{K}_{r}(x, t, \xi, \tau)$ have the form

$$
\mathbf{K}_{r}(x, t, \xi, \tau)=\left[k_{i j}^{r}(x, t, \xi, \tau)\right]_{i, j=1}^{N}=\left[k_{i j}^{r}(x, t) \bar{k}_{i j}^{r}(\xi, \tau)\right]_{i, j=1}^{N}, \quad r=1, \ldots, m,
$$

where $k_{i j}^{r}(x, t)$ and $\bar{k}_{i j}^{r}(\xi, \tau)$ are some functions, such that $k_{i j}^{r} \in L^{2}\left(0, T ; L^{2}\left(D_{r}\right)\right), \bar{k}_{i j}^{r} \in L^{2}\left(0, T ; H_{0}^{1}\left(D_{r}\right)\right)$.

Then the matrix $\left(\mathbf{K}_{r}(\xi, \tau, x, t)\right)^{T}$ entering in operator (2.7) is equal to

$$
\left(\mathbf{K}_{r}(\xi, \tau, x, t)\right)^{T}=\left[k_{j i}^{r}(\xi, \tau, x, t)\right]_{i, j=1}^{N}=\left[k_{j i}^{r}(\xi, \tau) \bar{k}_{j i}^{r}(x, t)\right]_{i, j=1}^{N}
$$

and $\forall \boldsymbol{\psi}=\left[\begin{array}{c}\psi_{1} \\ \vdots \\ \psi_{N}\end{array}\right] \in L^{2}\left(0, T ; L^{2}\left(D_{r}\right)^{N}\right)$, we have

$$
\begin{gathered}
\mathbf{P}_{r} \boldsymbol{\psi}(x, t)=\int_{0}^{T} \int_{D_{r}}\left(\mathbf{K}_{r}(\xi, \tau, x, t)\right)^{T} \boldsymbol{\psi}(\xi, \tau) d \xi d \tau \\
=\int_{0}^{T} \int_{D_{r}}\left[\begin{array}{ccc}
k_{11}^{r}(\xi, \tau, x, t) & \ldots & k_{N 1}^{r}(\xi, \tau, x, t) \\
\vdots & \ddots & \vdots \\
k_{1 N}^{r}(\xi, \tau, x, t) & \ldots & k_{N N}^{r}(\xi, \tau, x, t)
\end{array}\right]\left[\begin{array}{c}
\psi_{1}(\xi, \tau) \\
\vdots \\
\psi_{N}(\xi, \tau)
\end{array}\right] d \xi d \tau \\
=\int_{0}^{T} \int_{D_{r}}\left[\begin{array}{ccc}
k_{11}^{r}(\xi, \tau) \bar{k}_{11}^{r}(x, t) & \ldots & k_{N 1}^{r}(\xi, \tau) \bar{k}_{N 1}^{r}(x, t) \\
\vdots & \ddots & \vdots \\
k_{1 N}^{r}(\xi, \tau) \bar{k}_{1 N}^{r}(x, t) & \ldots & k_{N N}^{r}(\xi, \tau) \bar{k}_{N N}^{r}(x, t)
\end{array}\right]\left[\begin{array}{c}
\psi_{1}(\xi, \tau) \\
\vdots \\
\psi_{N}(\xi, \tau)
\end{array}\right] d \xi d \tau \\
=\left[\begin{array}{c}
\sum_{l=1}^{N} c_{l, 1} \bar{k}_{l 1}^{r}(x, t) \\
\vdots \\
\sum_{l=1}^{n} c_{l, N} \bar{k}_{l N}^{r}(x, t)
\end{array}\right] \in L^{2}\left(0, T ; H_{0}^{1}\left(D_{r}\right)^{N}\right)
\end{gathered}
$$

where

$$
c_{l, s}=\int_{0}^{T} \int_{D_{r}} k_{l, s}^{r}(\xi, \tau) \psi_{s}(\xi, \tau) d \xi d \tau, \quad l, s=1, \ldots, N, \quad r=1, \ldots, m
$$

\section{Acknowledgments}

This research was supported by Taras Shevchenko National University of Kyiv (Project 011U002529).

\section{REFERENCES}

1. J. Rauch, Hyperbolic Partial Differential Equations and Geometric Optics. //http://www.math.lsa.umich.edu/ rauch/nlgonotes.pdf

2. H. Gajewski, K. Gröger, K. Zacharias, Nichtlineare Operatorgleichungen und Operatordifferentialgleichungen, Akademie-Verlag; Berlin, 1974.

3. S. Mizohata, The theory of partial differential equations, Cambridge University Press, 1973.

4. J.L. Lions, Optimal control of systems described by partial differential equations, Springer- Verlag, New York, Heidelberg, Berlin, 1971.

5. Y. Podlipenko, Y. Shestopalov, Mixed Variational Approach to Finding Guaranteed Estimates for Solutions and Right-Hand Sides of the Second-Order Linear Elliptic Equations under Incomplete Data, Minimax Theory and its Applications, vol. 1, no. 2, pp. 197-244, 2016. 
6. O.G. Nakonechnyi, Minimax State Estimates for Abstract Neumann Problems, Minimax Theory and its Applications, vol. 3, no. 1, pp. 1-21, 2018.

7. M. Luz, M. Moklyachuk, Minimax-robust filtering problem for stochastic sequences with stationary increments and cointegrated sequences. Statistics, Optimization and Information Computing, vol. 2, no 3, pp. 176-199, 2014.

8. M. Luz, M. Moklyachuk, Minimax interpolation problem for random processes with stationary increments. Statistics, Optimization and Information Computing, vol. 3, no 1, pp. 30-41, 2015.

9. M. Moklyachuk, Minimax-Robust Estimation Problems for Stationary Stochastic Sequences. Statistics, Optmization and Information Computing, vol. 3, no. 4, pp. 348-419, 2015.

10. O. A. Kapustian, O. G. Nakonechnyi, The Minimax Problems of Pointwise Observation for a Parabolic Boundary-Value Problem, Journal of Automation and Information Sciences, vol. 34, no. 5, pp.52-67, 2002.

11. Minimax Theory and its Applications

12. V. O. Kapustyan, O. A. Kapustian, Y. K. Podlipenko, Minimax estimates of functionals from time-periodic solutions of Newmann boundary-value problems for parabolic equations. (Ukrainian), Bulletin of the University of Kiev. Series: Physics and Mathematics, Issue 2, pp. 220-232, 2005.

13. Yu. Podlipenko, V. Golovach The minimax approach to the estimation problem of parameters of the linearized Navier-Stokes equation under incomplete data. (in Russian) Journal of Numerical and Applied Mathemayics, No. 3 (99), pp. 70-88, 2009.

14. V. Hutson, J.S. Pym, M.J. Cloud, Applications of functional analysis and operator theory, 2ed., Elsevier, 2005. 